TITLE PAGE

\title{
Interdependency of the maximum range of flexion-extension of hand metacarpophalangeal joints
}

Author Names

V. Gracia-Ibáñez, M. Vergara, J.-L. Sancho-Bru

Filiation

Dpto. de Ingeniería Mecánica y Construcción. Universitat Jaume I- Castelló, SPAIN

Corresponding author:

VERÓNICA GRACIA IBÁÑEZ

vgracia@uji.es

TEL. +34964728109

FAX +34 964728106

DEPARTAMENTO DE INGENIERÍA MECÁNICA Y CONSTRUCCIÓN,

AVDA. SOS BAYNAT, UNIVERSITAT JAUME I,

E12071 - CASTELLÓ, SPAIN.

Funding:

This work was supported by the Spanish Ministry of Economy and Competitiveness under

Grant [project DPI2014]; Universitat Jaume I under Grant [project P1-1B2013-33] 


\title{
Interdependency of the maximum range of flexion-extension of hand metacarpophalangeal joints
}

\begin{abstract}
Mobility of the fingers metacarpophalangeal joints depends on the posture of the adjacent ones. Current Biomechanical hand models consider fixed ranges of movement at joints, regardless of the posture, thus allowing for non-realistic postures, generating wrong results in reach studies and forward dynamic analyses. This study provides data for more realistic hand models. The maximum voluntary extension (MVE) and flexion (MVF) of different combinations of metacarpophalangeal joints were measured covering their range of motion. Dependency of the MVF and MVE on the posture of the adjacent metacarpophalangeal joints was confirmed and mathematical models obtained through regression analyses (RMSE $\left.7.7^{\circ}\right)$.
\end{abstract}

Keywords: Interdependent limits, finger metacarpophalangeal joint, range of movement, hand biomechanical models.

\section{INTRODUCTION}

Biomechanical models of hands have been used for different applications such as in the design of prosthetic hands, studying disabilities, rehabilitation and functional assessment, and for ergonomic product design (Armstrong et al., 2009; Endo et al., 2014; Fok and Chou, 2010; Harih and Tada, 2015; Hemami et al., 2016; Park et al., 2014; Peña-Pitarch et al., 2014; Sancho-Bru et al., 2011; Valero-Cuevas et al., 2000; van Nierop et al., 2008; Wu et al., 2010). They simulate segments, joints and other tissues (muscles, tendons, ligaments or even skin) and use ranges of mobility at each joint that cover the full range of angles for each joint, regardless of the posture of other joints. However, it is well known that the movements of nearby joints are coordinated (Engel et al., 1997; Jindrich et al., 2004; Kuo 
et al., 2006; Soechting and Flanders, 1997). Lang and Schieber (Lang and Schieber, 2004) found that, although both the anatomical structure of the hand and the neuromotor system that control the hand restrict the independence of human finger movements, the anatomical structure limits finger independence to a greater degree. The connections in the flexorextensor mechanism and the fact that each motor unit actuates more than one tendon makes it unavoidable that fingers move in a coordinated way, which promotes the existence of kinematic synergies in the hand (Rearick and Santello, 2002; Santello et al., 1998). Santello et al. (Santello et al., 1998) found a high correlation between joint flexion angles in grasping actions, especially between the closest metacarpophalangeal (MCP) joints, which decreased with the distance between them. Furthermore, a non-linear relationship between the flexion of the MCP and the proximal interphalangeal joints of each finger has been reported (Braido and Zhang, 2004).

Therefore, the flexion-extension movement of an MCP joint depends on the angle of the adjacent joints, and this dependency affects the maximal angles in flexion and extension achievable at a specific joint. However, the biomechanical models described in the literature lack this restriction, thereby allowing for highly non-realistic postures. This might generate incorrect results, especially for studying reach of buttons and controls in ergonomic design of tools (e.g. pressing power button of a drill while maintaining it grasped) and for forward dynamic analyses. The aim of this work is to propose models for the interdependent MCP flexion-extension ranges of movement of the fingers based on experimental data in order to provide more realistic ranges than those currently used for ergonomic design or biomechanical models. 


\section{METHODS}

Postures of maximum voluntary flexion (MVF) and extension (MVE) of the MCP joints of the four fingers were recorded using a videogrammetric technique (Sancho-Bru et al., 2014) that provides flexion-extension and abduction-adduction rotation angles for each MCP joint. Flexion and ulnar deviations were considered positive.

The experiment, approved by the University Ethical Committee, was performed in two phases: (1) MVE on sample S1 (22 subjects, 11 males and 11 females, 21 right-handed); and (2) MVF on sample S2 (26 subjects, 13 males and 13 females, 23 right-handed). All the participants (Table 1) were free of hand lesions or pathologies, were properly informed and gave their written consent. As only eight of the subjects from sample S1 were available at the moment the MVF experiment was performed, both samples S1 and S2 were checked to be comparable (no expectable differences in MVE and MVF between groups). In order to do it, the MVE of the MCP of the index and the little fingers were measured (separately) and the samples compared by means of two analyses of variance (ANOVAs), one for index MVE and the other for little MVE (dependent variable was the MVE angle, independent variable was the sample).

Insert Table 1 here

Firstly, a reference posture (considered as zero rotation angles) was recorded (Figure 1, posture R1). For MVE, three starting postures (Figure 1) were used: hand lying on a flat surface (R1), and grasping cylinders with a diameter of $65 \mathrm{~mm}(\mathrm{R} 2)$ and $35 \mathrm{~mm}(\mathrm{R} 3)$; while only the flat one (R1) was used for MVF. Maximum voluntary movements of specific fingers (maintaining the other fingers in the three starting postures) were recorded (Figure 
2): each of the four fingers moving individually (postures ai, bi, ci and di, with $\mathrm{i}=1$ to 4 ), two adjacent fingers moving (postures ei, fi and gi), three adjacent fingers moving (postures hi and ji) and four fingers moving simultaneously (postures ki). Subindex 1 is used for MVE from the flat starting posture R1 (see figure 1), 2 for MVE from R2 posture, 3 for MVE from R3 posture, and 4 for MVF from R1. Movements of non-adjacent fingers were not considered because they are more difficult to perform and previous studies (Lang and Schieber, 2004; Santello et al., 2002, 1998) evidence that the closer the finger is, the more influence it has on the MVE/MVF. The cylinders were selected so that the range of extension of the MCP joints in the starting posture goes from $0^{\circ}$ (reference position) to approximately $90^{\circ}$ (cylindrical grasp with the cylinder with a diameter of $35 \mathrm{~mm}$ ), passing through an intermediate angle of approximately $45^{\circ}$ with the cylinder with a diameter of 65 $\mathrm{mm}$. In the case of MVF, special wooden pieces (Figure 2, images with subscript 4) were used to ensure that only the fingers involved in the desired movement flexed. This makes a total of 28 postures for MVE and 10 for MVF, covering a wide range of postures. The abduction angles of the MCP joints and the flexion of the interphalangeal joints were not controlled: each subject adopted the posture in which he/she achieved the MVF or MVE without any indications about the abduction posture. To obtain MVF and MVE values, the postures were maintained for 1 second and the average value of each record was considered.

Insert Figures 1 and 2 here

In the results, the MVE and MVF for each finger have been identified by adding a subindex: 2 for index, 3 for middle, 4 for ring, and 5 for little. 
Initially, the means across subjects for each joint were calculated for each of the 38 postures of Figure 2 in order to obtain representative statistics data to be compared with data reported in literature. These mean values across subjects were used only for this purpose, and all the data from all subjects was used for all the analyses described afterwards.

Twelve ANOVAs (four fingers $\mathrm{x}$ three starting postures) were performed with the data from all subjects to check whether MVE depended on the combination of fingers (dependent variable: MVE of each of the four joints; only factor: combination of fingers). Likewise, four more ANOVAs were performed for the dependent variable MVF. Each ANOVA was performed only with the data obtained while the finger being analysed was in MVF/MVE, either alone or in combination with others. As an example, for the index finger, the flat starting posture and MVE, the ANOVA was performed with the data for postures a1, e1, $\mathrm{h} 1$ and $\mathrm{k} 1$, the four possible combinations of fingers in which the index finger moves; for the middle finger, the flat posture and MVF, the ANOVA was performed with the data for postures b4, e4, f4, h4, j4 and k4; and so on. For those cases where significant differences were detected, a post-hoc test was performed (Tukey HSD with $\mathrm{p}=0.05$ ). Normality of distributions (Kolmogorov-Smirnov test), homogeneity of variances and homoscedasticity (Levene test) were checked previously.

Furthermore, to check the amount of the variability of the data attributable to the subjects, eight univariate ANOVAs (4 fingers x 2 movements -extension and flexion-) were performed with factors 'subject' and 'combination of fingers' for flexion, and 'subject', 'combination of fingers' and 'starting position' for extension. The F-ratios of the factors were compared. 
Eight linear regression analyses were performed with all the data from all subjects to obtain the desired models (dependent variable: the MVF/MVE of each of the 4 joints; independent variables: the flexion angles of all other MCP joints). Each linear regression analysis was performed only with the data obtained while the finger being analysed was in MVF/MVE, either alone or in combination with others, considering the motions from all starting positions. As an example, the analysis of MVE for the ring finger was performed with data from postures $\mathrm{c} 1, \mathrm{c} 2, \mathrm{c} 3, \mathrm{f} 1, \mathrm{f} 2, \mathrm{f3}, \mathrm{g} 1, \mathrm{~g} 2, \mathrm{~g} 3, \mathrm{~h} 1, \mathrm{~h} 2, \mathrm{~h} 3, \mathrm{j} 1, \mathrm{j} 2, \mathrm{j} 3$ and $\mathrm{k} 1$, the dependent variable being the angle of the MCP of the ring finger and the independent variables were the MCP angles of the other three fingers. All the analyses were performed using IBM SPSS Statistics 22 (IBM Corp. (C).

Finally, a verification experiment was developed to check the effectiveness of using the regression equations for two subjects that did not participate in the previous experiment. The subjects were a man (age: 49 years, hand length $184 \mathrm{~mm}$, corresponding to percentile $\mathrm{P}_{73}$ of all the participants, and breadth $87 \mathrm{~mm}, \mathrm{P}_{78}$ ) and a woman (age: 41 years, hand length $170 \mathrm{~mm}, \mathrm{P}_{31}$ of all the participants, and breadth $\left.76 \mathrm{~mm}, \mathrm{P}_{27}\right)$. They were asked to perform the MVFs/MVEs in 10 postures (Figure 3), six from the previous experiment (three MVF and three MVE) and four others inspired by the American Sign Language, attempting to achieve MVE/MVF of some of the fingers while keeping the others in a comfortable posture. With these equations, different estimations were made for MVF $\left(\mathrm{MVF}_{5}\right.$ in postures $\mathrm{A}$ and $\mathrm{C} ; \mathrm{MVF}_{2}, \mathrm{MVF}_{3}$ and $\mathrm{MVF}_{4}$ in postures $\mathrm{B}$ and $\mathrm{C}$ ) and for MVE $\left(\mathrm{MVE}_{1}\right.$ in postures $\mathrm{D}, \mathrm{E}, \mathrm{F}, \mathrm{H}$ and $\mathrm{K} ; \mathrm{MVE}_{3}$ and $\mathrm{MVE}_{4}$ in postures $\mathrm{E}$ and $\mathrm{F}$; and $\mathrm{MVE}_{5}$ in postures $\mathrm{F}$ and $\mathrm{G}$ ). The root mean square error (RMSE) of the differences between the measured MVFs/MVEs (with the videogrammetric technique) and their estimations from 
the regression equations were calculated.

Insert Figure 3 here

\section{RESULTS}

The ANOVAs performed to check whether the samples were comparable showed no significant differences $(p=0.293$ for index MVE and $p=0.111$ for little finger MVE).

Table 2 shows descriptive statistics (maximum, minimum, mean and standard deviation (SD)) for the means across subjects of the MVF/MVE of the MCP joints. Little finger shows the maximal extension $\left(-37.3^{\circ}\right)$, followed by index finger $\left(-30.2^{\circ}\right)$, while maximal flexion corresponds to the ring finger $\left(89.8^{\circ}\right)$. The dispersions observed within each finger, which are bigger for MVE, are attributable to the posture of the other fingers.

Insert Table 2 here

The ANOVAs confirmed that MVF and MVE depend significantly on the combination of fingers involved $(p<0.05)$ in the four cases of MVF and in six of the 12 cases of MVE. Figures 4 and 5 show box plots for MVE and MVF respectively, ordered by mean value, for cases where the differences between the combinations of fingers are significant in the ANOVAs. The horizontal grey-scaled bars in the graphs represent homogeneous groups, i.e. combinations of fingers between which there is no statistically significant difference in MVE/MVF. For example, in the case of the MVE of the index finger and the starting posture R3 (graph at top left) there are significant differences between postures e 3 and h3, but there is no significant difference between posture a3 and the other two (e3 and h3).

Insert Figures 4 and 5 here 
The F-ratios (table 3) of the eight additional ANOVAs showed that the variability attributable to the subject was more than 8 times lower than that attributable to the posture of the other fingers (accounted by the other factors: combination of fingers and starting position).

Insert Table 3 here

Table 4 shows the coefficients of the regression equations obtained for the MVE and MVF of each joint, together with adjusted coefficients of determination (adj. R2). All the equations included the constant term so that they can be expressed as:

$$
\text { Estimated MVFn or MVEn }=\text { Constant }+\sum_{\forall j \text { except } n} B_{j} \times M C P_{j}
$$

where $M V F_{n}$ is the MVF of the MCP joint for digit $n, M V E_{n}$ is the MVE of the MCP joint for digit $n, M C P_{j}$ is the posture of MCP joint $j$ (considering flexion as positive and extension as negative) and Constant and $B_{j}$ are the coefficients shown in Table 3 . Note that the regressions are better (higher adj. R2 values) when the number of postures used is bigger: higher values for MVE than for MVF, and for intermediate fingers than for extreme fingers, where the number of postures used are more limited.

Insert Table 4 here

Finally, for the verification experiment, the RMSE obtained between the measured and the estimated MVF/MVE was $7.7^{\circ}$. 


\section{DISCUSSION}

The maximum values for the means of the MVE/MVF (negative for extension and positive for flexion) shown in Table 2 are similar to the values of ranges of mobility reported in the literature $\left(90^{\circ}\right.$ flexion, $30-40^{\circ}$ extension (Kapandji I.A., 2007)). The mean MVE/MVF values shown in Table 2 are highly variable, as can be observed from the SD values and the minimum and maximum values. This high variability is not attributable to subjects, as the means have been obtained across subjects, but is due to the different postures considered for the other fingers, as it is reinforced by the ANOVAs and the F-ratios obtained. The dispersion of the mean MVE values is much higher than that of the MVF values, thus implying a higher dependency on the extension range of movement than on the flexion one. This can be explained by the constraints introduced by the juncturae tendinum connecting the extensor tendons on the hand metacarpals (Lang and Schieber, 2004; Santello et al., 1998), which is not present for the case of the flexor tendons in the palmar side of the hand. All these evidences highlight the fact that the fixed limits on the ranges of motion of the MCP joints used in existing models in literature are non-realistic and that better estimates of the inter-dependability of adjacent MCP postures are needed. Moreover, this study evidences that the MVE/MVF for the MCP joints depends on the combination of fingers involved in the movement in most cases and, as can be observed from the magnitude of the regression coefficients, generally, the closer the finger is, the more influence it has on the MVE/MVF, in agreement with other studies (Lang and Schieber, 2004; Santello et al., 2002, 1998). Again, this can be explained by the existing connections (juncturae tendinum and intertendinous fascia) between adjacent tendons. This has biomechanical and ergonomics implications. For example, postures with extreme flexion/extension of a 
particular MCP joint not accompanied by adjacent joints should be avoided in order to prevent high stresses arising from tight connections. This can occur when the grasping of an object has to be maintained with some fingers while other finger or fingers have to perform another action such as pressing a control button. Looking at the regression equations it can be observed that all the signs of the adjacent fingers are positive and have the biggest coefficients. This means that MVF (positive sign in the equation) is increased when adjacent fingers are more flexed, while in the case of MVE (negative sign) is reduced when adjacent fingers are more flexed. I.e., fingers tend to move together to the same direction maintaining a maximum relative flexion between adjacent MCP joints, and this maximum relative posture between fingers seems to determine the MVE/MVF that adjacent fingers can achieve.

The different orientations of the juncturae tendinum of the extensor tendons of the fingers (Abdel-Hamid et al., 2013; von Schroeder and Botte, 2001) become in different constraints among fingers. Extension of the middle MCP joint is similarly constrained regardless of the posture of the other fingers, so that no significant dependency was observed on the combination of fingers involved for any of the reference postures. Oppositely, ring MCP joint showed significant dependency for all reference postures, little MCP joint for the two most flexed reference postures, and index MCP joint only for the most flexed reference posture. Highest differences in all cases were found between the case in which only one finger was extended while the other ones were kept fixed, and the case in which three adjacent fingers were extended while only one finger was kept fixed.

The juncturae tendinum of the extensor tendons of the fingers may also introduce MCP flexion constraints among fingers, depending on the orientation of the juncturae tendinum, 
as flexion requires excursion of the extensor tendon (von Schroeder and Botte, 2001). This orientation highly depends on the relative flexion between adjacent MCP joints. As a consequence, significant dependency on the combination of fingers involved was observed for flexion of all MCP joints.

The significant differences in Figures 4 and 5, modelled using the regression equations, are in accordance with the physiological constraints provided by the juncturae tendinum connecting the extensor tendons (Lang and Schieber, 2004; Santello et al., 1998). For example, the connection from the middle to the index tendon, according to physiological observation (Abdel-Hamid et al., 2013), inserts more proximally into the middle tendon than into the index one when both fingers present a similar MCP joint angle (as in posture R1). Such orientation tends to limit index MCP flexion. As the middle finger flexes with respect to the index, such orientation tends to get inverted and can even generate an extension limitation on the index $\mathrm{MCP}$ joint. This is why $\mathrm{MVF}_{2}$ only presented significant differences when starting from posture R1, for which maximal flexion of the index finger was much lower when moving alone than when accompanied by the middle finger and, alternatively, $\mathrm{MVE}_{2}$ presented significant differences only when starting from the most flexed posture, R3.

The main limitation of this work is that the proposed model has been obtained using a limited, although varied, number of postures and the postures of the interphalangeal joints were not controlled (any flexion at these joints would generate an extra excursion of the extensor tendon, and a smaller one for the flexor tendons, which could have a slight effect on the MVE/MVF of the MCP joints). The ranges of motion estimated with the models proposed should be considered, thus, as indicative limits of such ranges of motions with a 
small error (the error for the 2 subjects in the validation experiment was low).

Furthermore, estimation of MVF was performed using wooden pieces that restricted the motion of other fingers, as these postures can only be reached using such a restriction. In a similar way, for MVE cylinders were used, and the fingers were restricted by exerting a force squeezing them. This fact sets a limitation, but only when using these data for free movements, not for grasping objects, as the types of restrictions used in this work are present when the hand is using objects and therefore more appropriate for grasp analysis.

The regression models proposed for the MCP flexion-extension range of movements provided good estimations for subjects that did not belong to the samples used to obtain the regression equations and can be easily implemented in existing biomechanical models to provide more realistic ranges than those currently used. The proposed models can benefit the existing biomechanical models used for very different applications, such as for the study of reach in ergonomic design, but could also be useful as reference values in clinical or rehabilitation assessments.

Future work could address obtaining complementary regression models using nonrestricted starting postures, more appropriate for being used in realistic animation involving free finger movements.

\section{CONCLUSION}

This study has shown that the MVE/MVF for each MCP joint depends on the posture of MCP joints of the other fingers. Generally, the closer the finger is, the more influence its MCP joint angle has on the MVE/MVF. 
Mathematical models are provided for quantifying this interdependency, yielding good estimations. These estimates should be considered as indicative limits, as they could be slightly modified because of the effect of the position of the IP joints.

The models proposed could benefit existing biomechanical models, providing more realistic ranges for their application. The data provided could also be useful as reference values in clinical or rehabilitation assessments.

\section{ACKNOWLEDGMENT}

We are grateful to the Spanish Ministry of Economy and Competitiveness for funding through its project DPI2014-52095-P, as well as to the Universitat Jaume I through its project P1-1B2013-33, in which this research is partially included. We also thank the graduate student Lourdes Perez Valiente for her collaboration in data collection.

This article has no relevant Conflict of Interests. 


\section{NOMENCLATURE}

$\begin{array}{ll}\text { MCP } & \text { metacarpophalangeal } \\ \text { MVF } & \text { maximal voluntary flexion } \\ \text { MVE } & \text { maximal voluntary extension } \\ \text { ANOVA } & \text { analysis of variance } \\ \text { SD } & \text { standard deviation }\end{array}$




\section{REFERENCES}

Abdel-Hamid, G.A., El-Beshbishy, R.A., Aal, I.H.A., 2013. Anatomical variations of the hand extensors. Folia Morphol. 72, 249-257.

Armstrong, T.J., Best, C., Bae, S., Choi, J., Grieshaber, D.C., Park, D., Woolley, C., Zhou, W., 2009. Development of a Kinematic Hand Model for Study and Design of Hose Installation. Lect. Notes Comput. Sci. 5620, 85-94.

Braido, P., Zhang, X., 2004. Quantitative analysis of finger motion coordination in hand manipulative and gestic acts. Hum. Mov. Sci. 22, 661-678. doi:10.1016/j.humov.2003.10.001

Endo, Y., Tada, M., Mochimaru, M., 2014. Reconstructing individual hand models from motion capture data. J. Comput. Des. Eng. 1, 1-12. doi:10.7315/JCDE.2014.001

Engel, K.C., Flanders, M., Soechting, J.F., 1997. Anticipatory and sequential motor control in piano playing. Exp. Brain Res. 113, 189-199. doi:10.1007/BF02450317

Fok, K.S., Chou, S.M., 2010. Development of a finger biomechanical model and its considerations. J. Biomech. 43, 701-713.

Harih, G., Tada, M., 2015. Finite element evaluation of the effect of fingertip geometry on contact pressure during flat contact. Int. j. numer. method. biomed. eng. 31 . doi:10.1002/cnm.2712

Hemami, H., Tarr, E., Li, B., Krishnamurthy, A., Clymer, B., Dariush, B., 2016. Towards a Cybernetic Model of Human Movement. Mech. Eng. Res.

Jindrich, D.L., Balakrishnan, A.D., Dennerlein, J.T., 2004. Effects of keyswitch design and finger posture on finger joint kinematics and dynamics during tapping on computer keyswitches. Clin. Biomech. 19, 600-608. doi:10.1016/j.clinbiomech.2004.03.003

Kapandji I.A., 2007. The Physiology of the Joints, Volume 1: Upper Limb, 6e ed. Churchill Livingstone/Elsevier.

Kuo, P.-L., Lee, D.L., Jindrich, D.L., Dennerlein, J.T., 2006. Finger joint coordination during tapping. J. Biomech. 39, 2934-2942. doi:10.1016/j.jbiomech.2005.10.028

Lang, C.E., Schieber, M.H., 2004. Human finger independence: limitations due to passive mechanical coupling versus active neuromuscular control. J. Neurophysiol. 92, 280210.

Park, J., Seo, N. jin, Son, J., Kim, W., Cheong, J., 2014. Postural variation of hand precision grips by object size. J. Mech. Sci. Technol. 28, 1641-1651. doi:10.1007/s12206-014-0309-x

Peña-Pitarch, E., Falguera, N.T., Yang, J.J., 2014. Virtual human hand: model and kinematics. Comput. Methods Biomech. Biomed. Engin. 17, 568-79. doi:10.1080/10255842.2012.702864

Rearick, M.P., Santello, M., 2002. Force synergies for multifingered grasping: effect of predictability in object center of mass and handedness. Exp. Brain Res. 144, 38-49. doi:10.1007/s00221-002-1024-x

Sancho-Bru, J.L., Jarque-Bou, N.J., Vergara, M., Perez-Gonzalez, A., 2014. Validity of a 
simple videogrammetric method to measure the movement of all hand segments for clinical purposes. Proc. Inst. Mech. Eng. H. doi:10.1177/0954411914522023

Sancho-Bru, J.L., Pérez-González, A., Mora, M.C., León, B.E., Vergara, M., Iserte, J.L., Rodríguez-Cervantes, P.J., Morales, A., 2011. "Towards a Realistic and SelfContained Biomechanical Model of the Hand" In: Theoretical Biomechanics. InTech. doi:10.5772/816

Santello, M., Flanders, M., Soechting, J.F., 2002. Patterns of Hand Motion during Grasping and the Influence of Sensory Guidance. J. Neurosci. 22, 1426-1435.

Santello, M., Flanders, M., Soechting, J.F., 1998. Postural hand synergies for tool use. J. Neurosci. 18, 10105-10115.

Soechting, J.F., Flanders, M., 1997. Flexibility and repeatability of finger movements during typing: analysis of multiple degrees of freedom. J. Comput. Neurosci. 4, 29-46. doi:10.1023/A:1008812426305

Valero-Cuevas, F.J., Towles, J.D., Hentz, V.R., 2000. Quantification of fingertip force reduction in the forefinger following simulated paralysis of extensor and intrinsic muscles. J. Biomech. 33, 1601-1609.

van Nierop, O.A., van der Helm, A., Overbeeke, K.J., Djajadiningrat, T.J.P., 2008. A natural human hand model. Vis. Comput. 24, 31-44.

von Schroeder, H.P., Botte, M.J., 2001. Anatomy and functional significance of the long extensors to the fingers and thumb. Clin. Orthop. Relat. Res. 74-83.

Wu, J.Z., An, K.-N.N., Cutlip, R.G., Dong, R.G., 2010. A practical biomechanical model of the index finger simulating the kinematics of the muscle/tendon excursions. Biomed. Mater. Eng. 20, 89-97. 


\section{Figure Captions List}

Fig. 1 Reference postures: R1) hand lying on a flat surface (used as zero rotation angles), and R2) and R3) hand grasping cylinders with different diameters.

Fig. 2 Postures for MVE and MVF of MCP joints. The letters refers to the combination of moving fingers, and subindexes refer to the posture of the controlled fingers.

Fig. 3 MVE and MVF postures used in the verification experiment for the two subjects. From A) to F) $3 \mathrm{MVF}$ and $3 \mathrm{MVE}$ postures from the previous experiment. From G) to K) postures inspired by the American Sign Language.

Fig. 4 Box-plots of MVE for cases in which the differences between the combinations of fingers is statistically significant in the ANOVAs. Boxes represent interquartile ranges (IQR) and medians, while whiskers represent values that are within 1.5 IQR. Circles represent extreme values (out of 1.5 IQR) and stars represent outliers (out of 3 IQR). The horizontal grey-scaled bars represent homogeneous groups. $\mathrm{MVE}_{n}$ is the MVE of MCP joint for digit $n$. Combinations are shown on the horizontal axis with the codes of postures used in figure 2.

Fig. 5 Box-plots of MVF for cases in which the differences between the combinations of fingers is statistically significant in the ANOVAs. The horizontal grey-scaled bars represent homogeneous groups. $\mathrm{MVF}_{n}$ is the 
MVF of MCP joint for digit $n$. Combinations are shown on the horizontal axis with the codes of postures used in figure 2 . 


\section{Table Caption List}

Table $1 \quad$ Description of samples S1 and S2.

Table 2 Descriptive statistics of MVF/MVE for each MCP joint (mean values across subjects). Flexion angles have been considered as positive and extension angles as negative, both in MVF or MVE.

Table 3 F-ratios of the ANOVAs performed to check the variability attributable to the subject in comparison to that due to the posture of other fingers.

Table 4 Coefficients of the eight regression equations for the MVE and MVF of each MCP joint and adjusted coefficients of determination of each equation (adj.R2). Flexion is considered as positive and extension as negative. As an example:

$\mathrm{MVF}_{2}=49.837+0.264 * \mathrm{MCP}_{3}+0.016 * \mathrm{MCP}_{4}+0.039 * \mathrm{MCP}_{5}$ 


\section{FIGURES}

Fig. 1
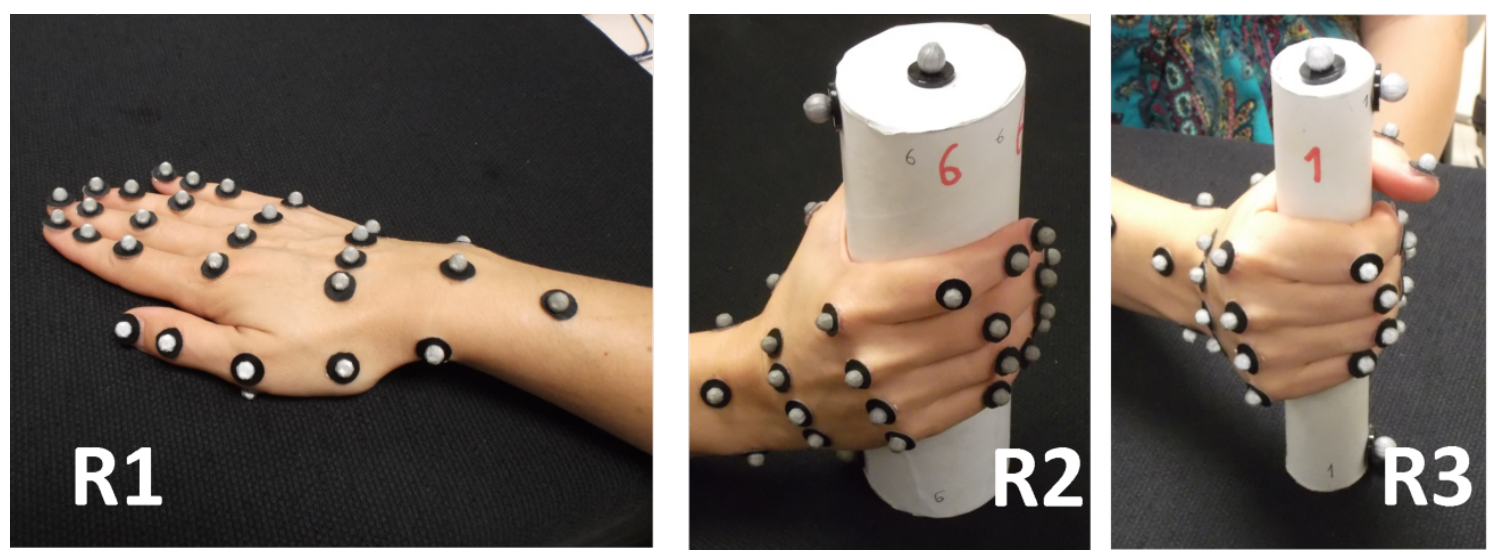
Fig. 2
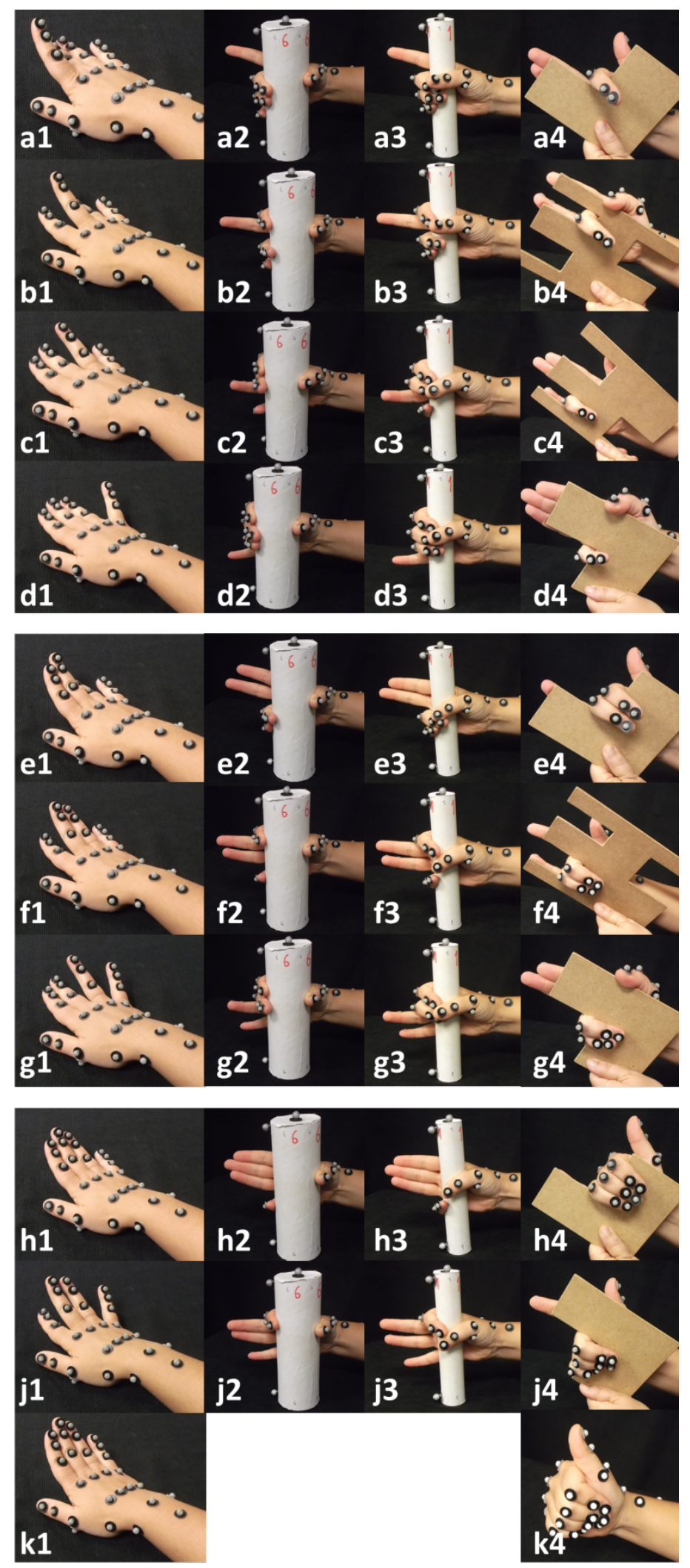
Fig. 3
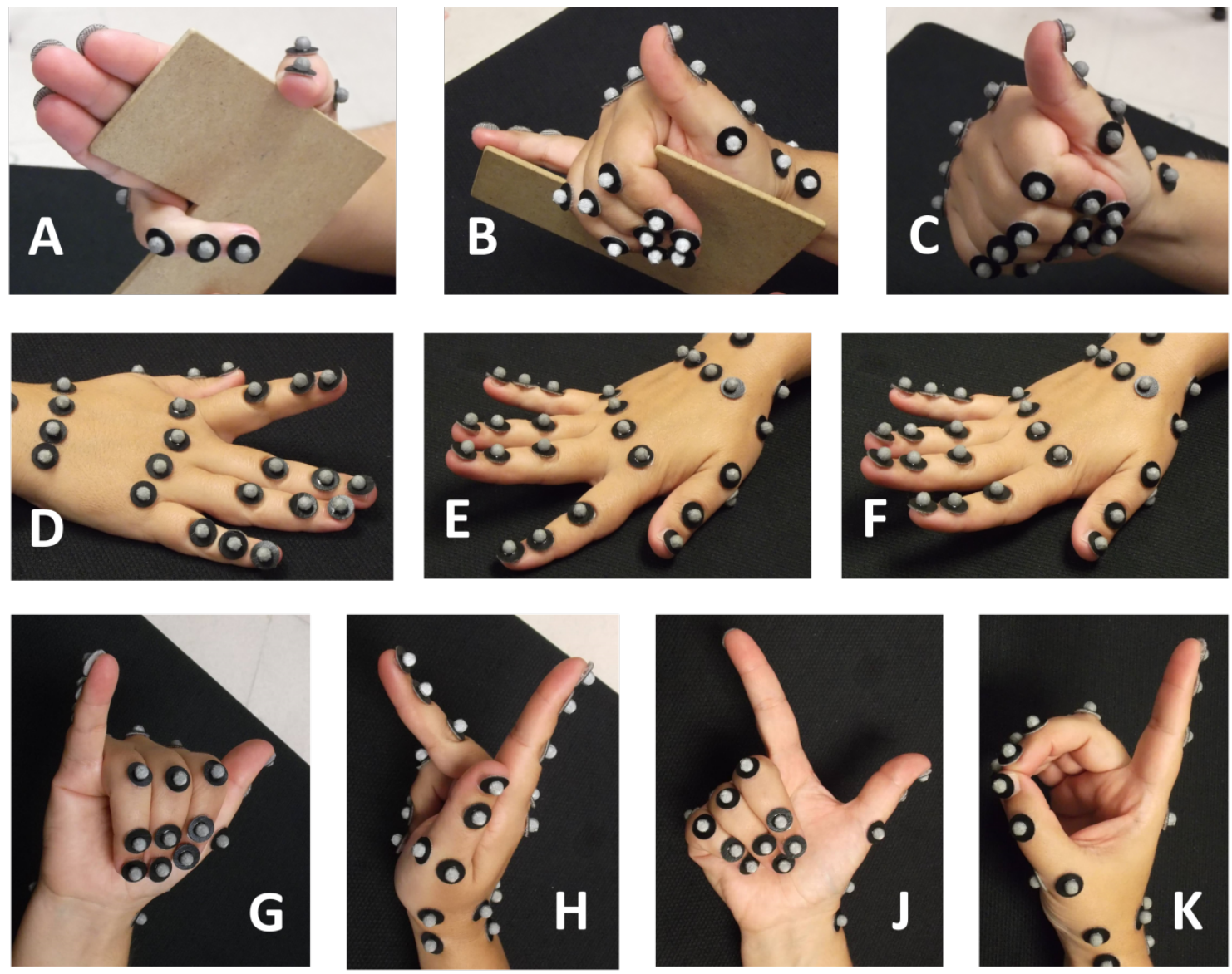
Fig. 4
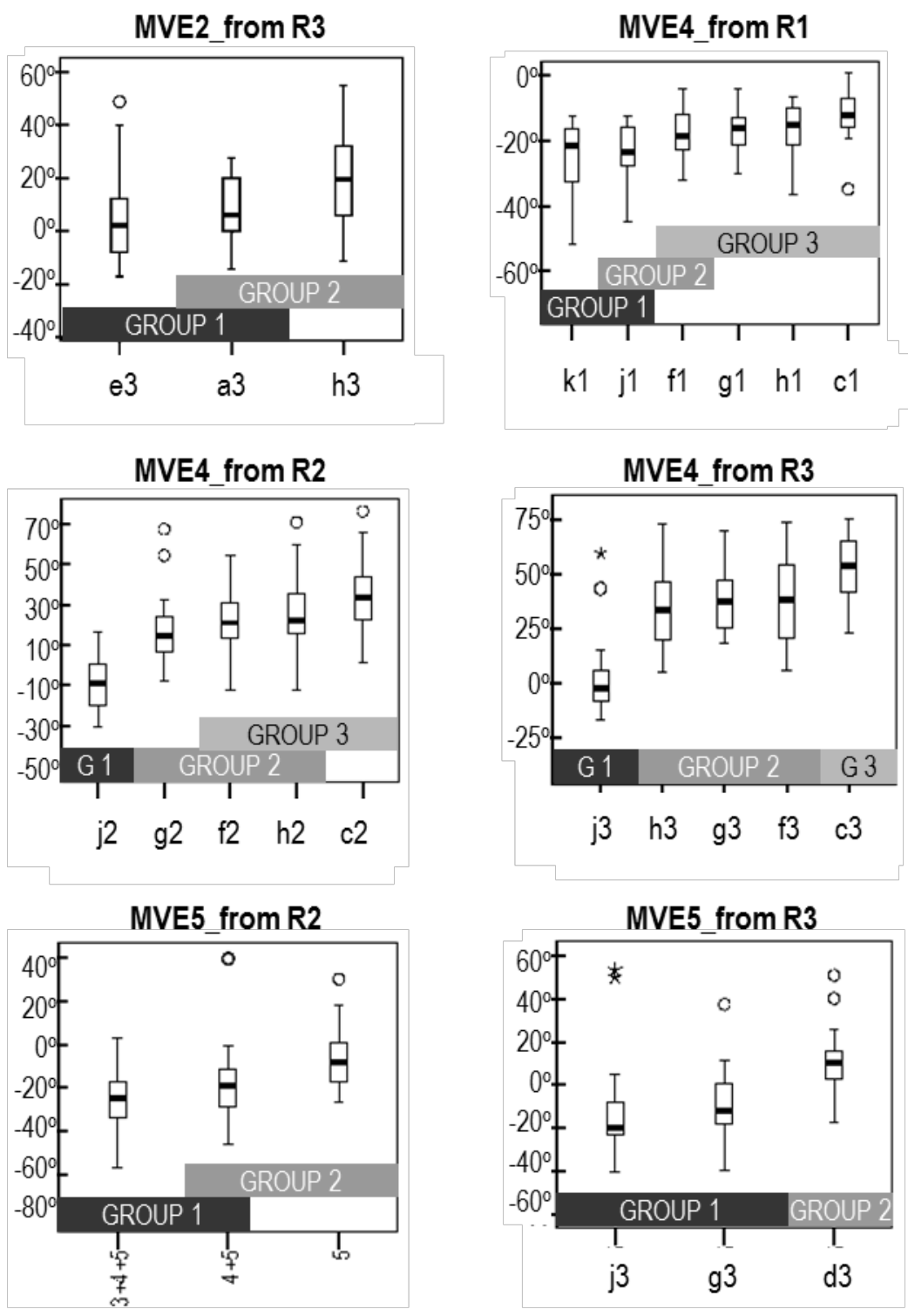
Fig. 5
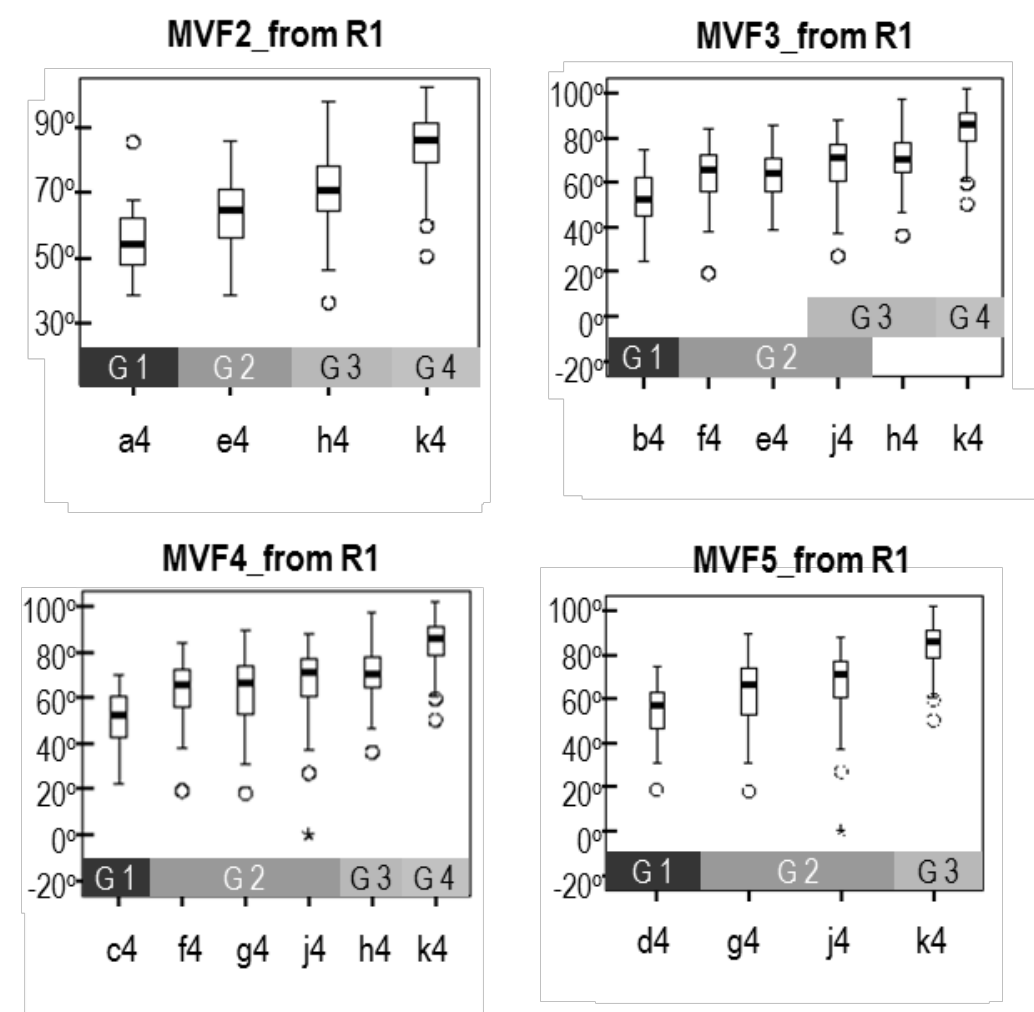


\section{TABLES}

Table 1

\begin{tabular}{cccc|ccc}
\hline & $\begin{array}{c}\text { Age } \\
\text { (years) }\end{array}$ & $\begin{array}{c}\text { S1 (MVE) } \\
\text { Hand } \\
\text { Length } \\
(\mathrm{mm})\end{array}$ & $\begin{array}{c}\text { Hand } \\
\text { Breadth } \\
(\mathrm{mm})\end{array}$ & $\begin{array}{c}\text { Age } \\
\text { (years) }\end{array}$ & $\begin{array}{c}\text { S2 (MVF) } \\
\text { Hand } \\
\text { Length } \\
(\mathrm{mm})\end{array}$ & $\begin{array}{c}\text { Hand } \\
\text { Breadth } \\
(\mathrm{mm})\end{array}$ \\
\hline Minimum & 24 & 151 & 68 & 23 & 155 & 72 \\
Maximum & 58 & 197 & 98 & 59 & 194 & 90 \\
Mean & 35.6 & 171.5 & 81.6 & 36.3 & 178.4 & 80.3 \\
SD & 9.7 & 12.5 & 8.2 & 9.5 & 10.2 & 5.4 \\
\hline
\end{tabular}


Table 2

\begin{tabular}{|c|c|c|c|c|c|}
\hline & & $\begin{array}{c}\text { Maximum } \\
\left({ }^{\circ}\right)\end{array}$ & $\begin{array}{c}\text { Minimum } \\
\left({ }^{\circ}\right)\end{array}$ & $\begin{array}{c}\text { Mean } \\
\left({ }^{\circ}\right)\end{array}$ & $\begin{array}{l}\text { SD } \\
\left({ }^{\circ}\right)\end{array}$ \\
\hline \multirow{4}{*}{ MVE } & MCP2 & 40.2 & -30.2 & -2.1 & 21.5 \\
\hline & MCP3 & 23.9 & -25.5 & -2.5 & 18.3 \\
\hline & MCP4 & 53.5 & -25.0 & 9.0 & 26.5 \\
\hline & MCP5 & 11.0 & -37.3 & -17.4 & 17.6 \\
\hline \multirow{4}{*}{ MVF } & MCP2 & 76.8 & 55.6 & 66.5 & 8.9 \\
\hline & MCP3 & 86.1 & 53.4 & 67.9 & 11.4 \\
\hline & MCP4 & 89.8 & 52.2 & 67.5 & 12.8 \\
\hline & MCP5 & 85.2 & 55.1 & 67.1 & 12.8 \\
\hline
\end{tabular}


Table 3

\begin{tabular}{|c|c|c|c|c|c|c|c|c|c|}
\hline & & \multicolumn{4}{|c|}{ MVE } & \multicolumn{4}{|c|}{ MVF } \\
\hline & & MCP2 & MCP3 & MCP4 & MCP5 & MCP2 & MCP3 & MCP4 & MCP5 \\
\hline \multicolumn{2}{|l|}{ Subject } & 8.5 & 13.0 & 8.4 & 6.5 & 9.2 & 9.5 & 7.0 & 4.8 \\
\hline \multirow{2}{*}{$\begin{array}{l}\text { Posture of } \\
\text { other fingers }\end{array}$} & $\begin{array}{l}\text { Combination } \\
\text { of fingers }\end{array}$ & 9.1 & 2.3 & 54.4 & 15.8 & \multirow[t]{2}{*}{50.2} & \multirow[t]{2}{*}{68.5} & \multirow[t]{2}{*}{59.8} & \multirow[t]{2}{*}{42.6} \\
\hline & $\begin{array}{l}\text { Starting } \\
\text { position }\end{array}$ & 205.4 & 371.5 & 476.1 & 92.6 & & & & \\
\hline
\end{tabular}


Table 4

\begin{tabular}{ccccccc}
\hline $\begin{array}{c}M V F_{n} / M V E_{n} \\
\left({ }^{\circ}\right)\end{array}$ & $\begin{array}{c}\text { Constant } \\
\left({ }^{\circ}\right)\end{array}$ & $B_{2}$ & $B_{3}$ & $B_{4}$ & $B_{5}$ & Adj. ${ }^{2}$ \\
\hline$M V F_{2}$ & 49.8 & & 0.264 & 0.016 & 0.039 & 0.516 \\
$M V F_{3}$ & 46.1 & 0.203 & & 0.264 & 0.014 & 0.705 \\
$M V F_{4}$ & 47.1 & 0.085 & 0.178 & & 0.253 & 0.697 \\
$M V F_{5}$ & 48.4 & 0.215 & -0.056 & 0.277 & & 0.501 \\
$M V E_{2}$ & -16.1 & & 0.316 & -0.074 & 0.332 & 0.618 \\
$M V E_{3}$ & -10.3 & 0.489 & & 0.419 & -0.065 & 0.846 \\
$M V E_{4}$ & 2.1 & 0.016 & 0.583 & & 0.384 & 0.897 \\
$M V E_{5}$ & -24.6 & 0.254 & -0.230 & 0.605 & & 0.613 \\
\hline
\end{tabular}

